\title{
Contemporaneous Infection of Dengue and Typhoid: 6 Months Study at Karachi
}

Sara Ahmed Kaleem ${ }^{1}$, Noor ul Ain ${ }^{1 *}$, Shireen Shahid ${ }^{1}$ and Abdullah Farhan Essa ${ }^{2}$

${ }^{1}$ Department of Serology, Dr. Essa Laboratory \& Diagnostic Centre, Karachi, Pakistan

${ }^{2}$ Department of Microbiology, Dr. Essa Laboratory \& Diagnostic Centre, Karachi, Pakistan

\begin{abstract}
Typhoid and Dengue both are so different diseases but they have some similar routine manifestations like fever. Our 6 months performed study was to determined contemporaneous infection of both diseases having same routine manifestations in patients at the same period of time. We were collected the blood samples from suspected patients. The serum were separated out through centrifugation from the desired blood samples and tested for typhoid by Rapid Immuno-chromatographic (typhidot) technique and for the dengue we performed NS1 Rapid technique. The performed results showed that $64.4 \%$ typhoid positive from dengue negative patients and $4 \%$ dengue positive from typhoid negative patients but we were not found any suspected patient who infected with both diseases at the same time. From our performed results we can conclude that the contemporaneous infections should be confirmed by diagnostic laboratory properly because it may leads to misdiagnosis and creates difficulties to the physician for their respective treatments. To restraint the contagious infections we should prevent the route of infections because prevention is better than cure.
\end{abstract}

Keywords: Fever; Typhoid; Dengue; Immuno-chromatographic; NS1

\section{Introduction}

Dengue and typhoid both infections represents fever with some other clinical manifestations. But the route of spreading infection is totally different with each other's. Route of transmission is fecal-oral route (by contaminated food and water) also the direct contact with infected person [1]. The dengue is transmitted by the infective female mosquito bite "Aedes albopictus" [2]. Causative agent of typhoid fever is "Salmonella typhi or paratyphi"; the clinical manifestation of the typhoid is fever with complaints like headache, diarrhea, vomiting, loss of appetite, nausea, malaise, slight dry cough, rose spot on chest, trunk and the back [3]. Dengue (Arbovirus) also known as break-bone fever. There are four serotypes involved in dengue named as DEN1 DEN2 DEN3 and DEN4, the clinical manifestation of the dengue is fever with complaints like headache, diarrhea, vomiting, loss of appetite, nausea, skin spots but the confirmatory symptoms is bleeding (avoid aspirin), decrease platelets count, low blood pressure (dangerously) [4,5]. Because of the same clinical manifestations it may create difficulties to misdiagnosis the diseases for a physician [6]. Our $6^{\text {th }}$ months performed study is tried to resolve the misdiagnosis of both diseases for the physician. Pakistan is the country in which the dengue is so contagious infection huge number of epidemics [7]. The duration of our performed study is from Dec 2016 to May 2017, the study area covered the Karachi city. Total population of the city is 23.5 million and this is also one of the common flood affected city.

\section{Materials and Methods}

In our performed study blood samples were collected in a gel tube from the disease suspected patients then the samples were allowed to centrifuge with the help of centrifugation machine at $4000 \mathrm{rpm}$ (rotation per minute) and were separated required serum from blood sample The serum samples were tested with their respective offer which is given by the physicians. For Dengue NS1 test we used panbio dengue early rapid kit with their sensitivity rate is $91.89 \%$ and specificity rate is $98.39 \%$ and for the typhoid we used TYPHIDOT Rapid IgM by reszOn diagnostics international with their specificity rate is $85.9 \%$ and sensitivity rate is $96.7 \%$ respectively (Figure 1 ).
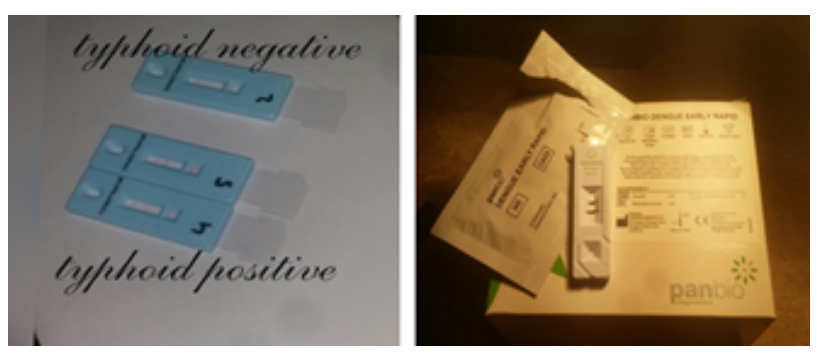

Figure 1: Panbio dengue early rapid kit.

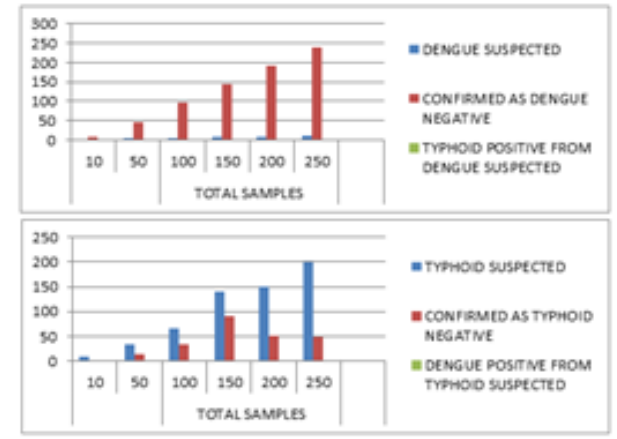

Figure 2: Samples showing the results of dengue and typhoid.

*Corresponding author: Noor ul Ain, Department of Serology, Dr. Essa Laboratory \& Diagnostic Centre, Karachi, Pakistan, Tel: +92341226346; E-mail: noorulain@ essalab.com

Received: July 17, 2017; Accepted: September 22, 2017; Published: September 28, 2017

Citation: Kaleem SA, Ain NU, Shahid S, Essa AF (2017) Contemporaneous Infection of Dengue and Typhoid: 6 Months Study at Karachi. J Infect Dis Med 2: 116. doi: $10.4172 / 2576-1420.1000116$

Copyright: (c) 2017 Kaleem SA, et al. This is an open-access article distributed under the terms of the Creative Commons Attribution License, which permits unrestricted use, distribution, and reproduction in any medium, provided the original author and source are credited. 


\section{Results}

We performed total 250 samples which is reported to the laboratory for the diagnosis of typhoid or dengue both the diseases. The positivity rate of the typhoid sample is $64.4 \%(161 / 250)$ the $4 \%(10 / 250)$ positivity rate for the dengue and rest of the samples showed negative results and there is no results found which having both typhoid and dengue positive as shown in Figure 2.

\section{Discussion}

In our $6^{\text {th }}$ months performed study we checked clinical manifestation of the two different infectious diseases which may cause misdiagnosis to the physicians for the treatment. We performed for the diagnosis of dengue we were done dengue NS1 and for the diagnosis of typhoid fever we were done typhidot (ICT Technique) [8]. Typhidot test is to check the presence of antibodies (IgM) of $S$. typhi or S. paratyphi in suspected serum samples [9]. The principle of the ICT kit is by the help of capillary action the immunological reaction is appeared on chromatographic paper which is visibly seen like bands. We found $64.4 \%$ typhoid positive patients from dengue negative samples and $4 \%$ dengue positive from typhoid negative samples but we were not found both dengue and typhoid positive at the same time. But from our related past paper the result were found like $3.40 \%$ numbers of patients were infected by both typhoid and dengue $5.81 \%$ dengue suspected patients were found negative for dengue but positive for typhoid. Again $0.18 \%$ numbers of typhoid suspected patients found negative for typhoid but positive for dengue [10]. So the patients must diagnosed properly by the physicians for the better cure.

\section{Conclusion}

During $6^{\text {th }}$ December 2016 to $31^{\text {st }}$ May 2017 we performed our study and we concluded that the physicians should kept in their mind before they give treatment to the patients that contemporaneous infections may leads to misdiagnosis and creates difficulties that's why the proper diagnosis is so important for both the infections either dengue or typhoid. Because of the unhygienic environment cause contagious infections like dengue or typhoid so people should clean our surroundings and dispose the waste and control the drainage of water because water logging helps mosquito breeding which increases the dengue infections. So the people take good care of their hygiene and clean the environment also. The government should take a step to aware peoples for their hygiene because the prevention is better than cure.

\section{Acknowledgements}

The researcher is very thankful for the cooperation of all the serological staff of Dr. Essa laboratory and diagnostic centre head office.

\section{References}

1. http://www.who.int/rpc/TFGuideWHO.pdf

2. Fabrizio C, Lepore L, Chironna M, Angarano G, Saracino A (2017) Dengue fever in travellers and risk of local spreading: case reports from Southern Italy and literature update. New Microbiol 40: 11-18

3. http://www.emedicinehealth.com/typhoid_fever_enteric_fever/page4_em.htm

4. https://en.wikipedia.org/wiki/Dengue_fever

5. http://www.medicinenet.com/dengue_fever/article.htm

6. Chrispal A, Boorugu H, Gopinath KG, Chandy S, Prakash JA, et al. (2010) Acute undifferentiated febrile illness in adult hospitalized patients: the disease spectrum and diagnostic predictors-an experience from a tertiary care hospital in South India. Tropical doctor 40: 230-234.

7. https://en.wikipedia.org/wiki/2011_dengue outbreak in Pakistan

8. https://www.slideshare.net/amerali6/immunochromatographic-assays

9. https://www.thermofisher.com/us/en/home/references/protocols/cell-andtissue-analysis/elisa-protocol/elisa-sample-preparation-protocols/plasma-andserum-preparation.html\#prot3

10. Mridul M, Manjit C, Sibarani T (2016) Concurrent infection of typhoid and dengue: A vice-versa study at Lakhimpur. Int J Curr Pharm Rev Res 7: 30-33. 